\title{
DIFERENCIAS ENTRE LA POBLACIÓN NATIVA Y LA MIGRANTE EN MONTERREY*
}

\author{
HaRley L. Browning y Waltraut Feindt \\ Population Research Center, \\ Universidad de Texas
}

\section{INTRODUCCIÓN}

El desarrollo económico y la modernización han sido rasgos característicos de la mayor parte de los países en el siglo xx. A este proceso lo acompaña una redistribución masiva de la población a la que llamamos urbanización, consistente en que una proporción creciente de la población total se localiza en las áreas urbanas. ${ }^{1}$

Existen grandes centros metropolitanos (100000 ó más), que frecuentemente acusan elevadas tasas de crecimiento demográfico. En América Latina son comunes incrementos superiores al $5 \%$, lo que se explica no sólo por las tasas de fecundidad muy elevadas, sino por la inmigración, que contribuye en términos generales con un 40 a $60 \%$ del crecimiento total. La inmigración neta proviene en su mayor parte de fuentes internas.

Algunos de los centros metropolitanos más grandes en América Latina (Buenos Aires, São Paulo y Caracas, entre otros) han tenido, en ocasiones, corrientes importantes de inmigrantes, en su mayor parte de Europa. En años recientes, sin embargo, esta corriente se ha reducido mucho, por lo que la inmigración efectivamente puede considerarse como de origen interno. La mayor parte del sector rural, que representa alrededor de la mitad de la población total, ${ }^{2}$ practica aún la agricultura tradicional. Como consecuencia, aunque los grandes centros metropolitanos atraen migrantes de comunidades de dis-

* Esta investigación se basa en un proyecto patrocinado conjuntamente por el Centro de Investigaciones Económicas, Facultad de Economía de la Universidad de Nuevo León, y el Population Research Center, Departamento de Sociología, de la Universidad de Texas. El proyecto fue dirigido por Jorge y Elizabeth Jelin Balán, en un principio adscritos a la Universidad de Nuevo León, y Harley L. Browning. Agradecemos a ellos y a Alvan O. Zárate su valiosa crítica. Nuestro agradecimiento también a Richard Rochwell y Lee Litzler por su asesoramiento estadístico y su asistencia en la programación. La traducción del inglés fue hecha por José B. Morelos, de El Colegio de México.

1. Las publicaciones más recientes sobre este tópico son Philip M. Hauser y Leo F. Schnore, comps., The Study of Urbanization, Nueva York, John Wiley \& Sons, 1965, y Kingsley Davis, "The Urbanization of the Human Population", Cities, Nueva York, Alfred A. Knopf, 1965, pp. 3-24. Sobre América Latina, véase Philip M. Hauser, comp., Urbanization in Latin America, París, Unesco, 1961.

2 Véase, por ejemplo, Gideon Sjoberg, "Rural-Urban Balance and Models of Economic Development", en Neil J. Smelser y Seymour Martin Lipset, comps., Social Structure and Mobility in Economic Development, Chicago, Aldine, 1966, pp. 235-261. 
tantes clases y tamaño, una gran proporción de los mismos tiene origen rural. Esto significa que la composición social de la población de un área metropolitana es muy heterogénea.

El propósito de este trabajo es investigar la heterogeneidad de la población masculina entre los 21 y los 60 años de edad con base en información obtenida en una encuesta llevada a cabo en Monterrey en 1965. La dimensión nativo-migrante se examinará con relación a cuatro indicadores socioeconómicos : educación, ocupación, ingreso y vivienda.

Las distintas características de Monterrey constituyen en sí una razón suficiente para un estudio de esta naturaleza. ${ }^{3}$ México ha generado y sostenido un fuerte impulso económico durante los últimos veinticinco años. A su vez la expansión económica de Monterrey ha sobrepasado a la del país en su conjunto. Además, es el segundo centro industrial, después de la ciudad de México, y el principal productor de hierro y acero. El crecimiento de la población de Monterrey ha sido excepcional. Entre 1940 y 1965, la población de su área metropolitana se quintuplicó al pasar de aproximadamente 186000 a un total estimado de 950000.4 Cerca de la mitad de su crecimiento medio anual de $6 \%$ se debe a la inmigración neta.

Por basarse este estudio en una sola área metropolitana de un país, en un período histórico dado, surge la pregunta de si estos resultados pueden generalizarse a otras situaciones. Nosotros sugerimos que las diferencias nativo-migrante no son constantes en el espacio ni en el tiempo, y que su comportamiento varía de acuerdo con un número de factores. Entre ellos: 1) el nivel y la tasa de desarrollo económico del país; 2) el nivel y la tasa de urbanización; 3 ) las diferencias de nivel de vida entre el área urbana y la rural; y 4) la tasa de crecimiento del área metropolitana.

En la muestra representativa de Monterrey, el $56 \%$ de los migrantes se crió en áreas rurales y más de la mitad de estos migrantes de origen rural llegaron ( $\sin$ etapas intermedias) directamente a Monterrey. Otro $21 \%$ creció en pequeñas localidades (5000 a 20000) en que los servicios sociales y generales son frecuentemente escasos. Apenas el $5 \%$ de los migrantes se formó en localidades (de 100000 y más) comparables a Monterrey. Este comportamiento, en el que una gran proporción de migrantes tiene origen rural o de pequeñas localidades, difiere en forma marcada del registrado en los países altamente desarrollados y urbanizados, como Estados Unidos, donde la mayoría de los migrantes de las áreas metropolitanas proceden de

3 A partir del último decenio se han efectuado importantes estudios sobre los migrantes a las grandes ciudades de América Latina. Además de los estudios de Gino Germani sobre Buenos Aires, José Matos Mar sobre Lima, Andrew Pearse sobre Río de Janeiro, y Rubens Brandão Lopes sobre São Paulo, publicados en Hauser, op. cit., existen también Hutchinson y otros, Mobilidade e Trabalho: um estudo na cidade de São Paulo, Río de Janeiro, Centro Brasileiro de Pesquisas Educacionais, 1960; y Juan C. Elizaga, "A Study of Migration to Greater Santiago (Chile)", Demography, Vol. 3, Núm. 2, 1966, pp. 352-377. Limitaciones de espacio no nos permiten realizar comparaciones directas y sistemáticas de los resultados de estos estudios con los obtenidos en el presente artículo.

4 Agradecemos al profesor Romeo Madrigal, del Centro de Investigaciones Económicas, Facultad de Economía, Universidad de Nuevo León, la estimación de la población en 1965. 
otras áreas metropolitanas. En tales condiciones, la distinción nativomigrante tendría muy poca importancia porque los antecedentes de los dos grupos diferirían relativamente poco.

La tasa de crecimiento de la población del área metropolitana está relacionada con las proporciones que los migrantes y nativos representan en la población metropolitana total. Cuanto mayor sea la tasa de crecimiento, mayor será la proporción de los migrantes en el total. Las tasas de crecimiento son también un indicio de la "flexibilidad de la estructura ocupacional" de la metrópoli. Un crecimiento lento es reflejo de un bajo desarrollo económico, y en tal caso los migrantes se enfrentan a grandes dificultades para adaptarse al medio ambiente, y en especial para competir con los nativos.

Para resumir nuestras consideraciones sobre la extensión general de los resultados de Monterrey, creemos que el comportamiento descrito se encontrará en otras áreas metropolitanas de rápido crecimiento tales como la ciudad de México, Guadalajara y algunas ciudades fronterizas. El comportamiento general también se encontraría en otras ciudades de veloz crecimiento en América Latina que tengan un nivel de desarrollo económico comparable. Se desconoce si este comportamiento podría darse en ciudades en expansión vertiginosa de Africa y Asia. No es de esperar que el comportamiento de Monterrey se encuentre en los países altamente urbanizados e industrializados.

\section{La CLASIFICACIÓN EN CATEgORÍAS MigRatoRIaS}

Como ya se mencionó, el principal objeto de este trabajo es determinar, respecto a la población adulta masculina de Monterrey, el efecto de la categoría migratoria sobre importantes variables socioeconómicas. ¿Cómo diferenciar la población? La manera convencional consiste en utilizar la distinción nativo-migrante, que es la que existe en casi todos los censos y en la mayor parte de las encuestas. Si una persona es entrevistada en un lugar distinto a su lugar de nacimiento, se le clasifica como migrante, y si nació en la comunidad se le clasifica como nativa. ${ }^{5}$ Pero esta distinción se hace sólo con base en el lugar de nacimiento. Lo que nos interesa es cuánto dura la "exposición" al medio ambiente de Monterrey metropolitano ; ${ }^{6}$ por consiguiente la diferenciación sobre la base arriba mencionada dista de ser ideal. Por ejemplo, los migrantes que llegaron a Monterrey una semana antes de ser enumerados caerían en la misma categoría que los

5 La terminología varía, a medida que algunos investigadores prefieren utilizar "no migrante" en lugar de nativo. En realidad, un número apreciable de personas clasificadas como no migrantes migran bajo la forma de migración de regreso.

6 Anticipándonos a las críticas tal vez deberíamos modificar de forma tal que se lea "duración de exposición potencial" por el hecho de que la gente que está físicamente en el medio ambiente urbano no necesariamente está expuesta a las instituciones y actividades urbanas. Oscar Lewis, con su noción de "campesinos urbanos" "Urbanization Without Breakdown: a case study", Scientific Monthly, LXXV, julio de 1952, pp. 3141) y Herbert Gans, en su The Urban Villagers (Nueva York, Free Press, 1962) han puesto en duda explícitamente el supuesto de igual exposición, sobre todo con relación a áreas urbanas grandes y complejas. Este aspecto no puede considerarse en detalle en este documento, aunque más tarde se tocarán ciertos aspectos del mismo. 
que llegaron 40 años antes. Para tener en cuenta este factor, la categoría de migrante se dividirá en tres grupos, tomando como base su período de residencia en Monterrey: de 0-9 años, 10-19 años y 20 años y más.

Comenzando por la categoría de nativos, la exposición a Monterrey puede analizarse en términos del lapso de vida del entrevistado y del de sus padres. Por lo tanto, se distinguirá entre las categorías de nativos de primera y de segunda generación. La primera generación de nativos será aquella cuyos padres nacieron fuera de Monterrey, mientras que la segunda abarcará a aquellos de los que al menos uno de los padres nació y creció en Monterrey. Como ambas generaciones de nativos se formaron en Monterrey, la distinción está realmente asociada al contexto familiar en que se desarrolla. Es de esperar que la segunda generación de nativos esté más adaptada a la vida de Monterrey porque sus "raíces" son más profundas, por ser al menos uno de sus padres nativos de la metrópoli. En cambio, es común que la primera generación de nativos tenga padres con origen rural o de pequeñas localidades. (En la muestra representativa de Monterrey esto ocurre en aproximadamente el $80 \%$ de los padres.) Es de suponer que estos padres tienen menos posibilidades de proporcionar a su descendencia una preparación y orientación generales que pudieran serles útiles en su éxito en Monterrey.

Con estas divisiones se ha ampliado la dicotomía original de nativos y migrantes a cinco grupos. Sin embargo, al considerar los migrantes hemos olvidado una característica importante. Los hemos clasificado por años vividos en Monterrey, pero no hemos considerado la etapa correspondiente en el ciclo vital del entrevistado, al llegar éste a Monterrey. Tomemos un ejemplo: una persona pudo haber nacido fuera de Monterrey, digamos en el área rural, pero llegó a la ciudad a los pocos meses de edad. Si se considera que toda su experiencia individual como ser humano ha transcurrido dentro de la ciudad, parecería poco razonable que se le clasificase como migrante aun cuando quedaría en esta categoría si se utilizase el lugar de nacimiento. Por lo tanto, conviene distinguir entre migrantes, nativos por nacimiento $\mathbf{y}$ nativos por adopción. Estos últimos son los que han nacido fuera de la comunidad pero han pasado en ella la mayor parte de sus años de formación, que en nuestro caso comprende el período entre los 5 y los 15 años de edad, período durante el cual el individuo adquiere su orientación básica en el mundo extrafamiliar, así como también los fundamentos de su educación formal. La distinción entre nativos por nacimiento y nativos por adopción requiere, además, establecer la distinción entre la comunidad de nacimiento y la de origen; esta última es aquella en que el individuo ha pasado la mayor parte de su formación educativa, es decir, entre los 5 y los 15 años. ${ }^{7}$

7 En su estudio de movilidad en Oakland, Lipset y Bendix preguntaron a los entrevistados en dónde habían vivido la mayor parte de su tiempo entre los 13 y los 19 años de edad (Seymour Martin Lipset y Reinhord Bendix, Social Mobility in Industrial Society, Berkeley y Los Angeles, University of California Press, 1959 , p. 204). Blau y Duncan preguntaron en su muestra representativa de la población masculina norteamericana de 20 a 64 años, por el tamaño de la localidad en que habían vivido a la edad de 16 años (Peter M. Blau y Otis Durley 
Con esto tenemos seis categorías migratorias, que se ordenan según la duración de la exposición a Monterrey y se identifican por las letras $A$ a $F$ :

\section{Categoría migratoria}

$A$ Migrantes con período corto de exposición

$B$ Migrantes con período intermedio de exposición

$C$ Migrantes con período largo de exposición

$D$ Nativos por adopción

$E$ Nativos de primera generación

$F$ Nativos de segunda generación

\section{Criterios}

No nacidos en la ciudad, ni formados en ella; han vivido menos de diez años en la misma

No nacidos en la ciudad, ni formados en ella; pero han vivido de 10 a 19 años en la misma

No nacidos en la ciudad; tampoco han pasado en ella sus años de formación, pero han vivido 20 o más años en la ciudad

Nacidos fuera de la ciudad, pero los años de formación transcurrieron en ella

Nacidos en la ciudad; ninguno de los padres ha nacido en ella

Nacidos en la ciudad; cuando menos uno de los padres ha nacido también en la ciudad

Algunas características de esta clasificación merecen comentarse. Primero, es evidente que la "exposición" no es un fenómeno unidimensional susceptible de derivarse de una variable, como, por ejemplo, el número de años vividos en la ciudad. Para establecer esta clasificación se utilizaron cuatro variables: 1) lugar de nacimiento del individuo; 2) lugar de nacimiento de los padres; 3 ) etapa en el ciclo vital del individuo en la que se inicia el contacto con la ciudad; 4) número de años durante los cuales el migrante ha vivido en Monterrey. El número medio de años vividos en Monterrey ${ }^{8}$ para las categorías migratorias es la siguiente:

$\begin{array}{lccccc}A & B & C & D & E & F \\ 5.7 & 15.1 & 29.3 & 31.3 & 36.9 & 37.8\end{array}$

Debe puntualizarse que algunos nativos por adopción pueden tener menos de 20 años de residencia en la ciudad, aunque como grupos están clasificados en un grado más alto de exposición que los migrantes con período largo de exposición.

Finalmente, es necesario subrayar que no estamos considerando en la clasificación la experiencia residencial de los migrantes antes de

Duncan, The American Occupational Structure, Nueva York, John Wiley \& Sons, 1967 , p. 447). Considerando que muchos jóvenes mexicanos ya habrán abando nado la escuela e ingresado a la fuerza de trabajo al llegar a esta edad, utilizamos un grupo de edad menor, 5-15.

8 Las medias de $E$ y $F$ no son simplemente la edad del hombre. Reflejan también el tiempo que pasaron en la ciudad ya que se dedujo cualquier período de ausencia menor de seis meses de Monterrey. 
incorporarse a Monterrey, ni de los nativos que hayan vivido fuera en algún momento. Esta experiencia sin duda alguna puede afectar, como en efecto sucede, sus oportunidades de vida en Monterrey. Incorporar formalmente este factor en el esquema de clasificación requeriría una ampliación de las categorías y más adelante complicaría el análisis. Deberá recalcarse que cerca de las tres cuartas partes de los migrantes a Monterrey tienen origen rural y de pequeñas localidades, y que sólo el $5 \%$ proviene de áreas metropolitanas. Por lo tanto, el hacer a un lado el origen de los migrantes no tendrá la importancia que podría tener en otras localidades.

La prueba real de cualquier esquema de clasificación es cómo opera. Las seis categorías migratorias serán probadas frente a las siguientes variables socioeconómicas: educación, ocupación, ingreso y vivienda. La hipótesis básica es la siguiente: a mayor duración de exposición al medio ambiente urbano, mayores serán los niveles que alcancen cada una de las variables socioeconómicas. Utilizando caracteres alfabéticos para identificar los seis grupos podemos esperar que:

$$
A<B<C<D^{\circ}<E<F
$$

Independientemente de si existe un continuum nativo-migrante se presenta un "espaciamiento" entre los seis grupos. Si no existe un espaciamiento parejo, ¿puede afirmarse que existe una "discontinuidad" significativa que divida los seis grupos en una dicotomía natural que justificaría la distinción común entre nativos y migrantes? De existir una o más discontinuidades, ¿dónde se presentan?

\section{LOS DATOS}

Los datos utilizados en este estudio provienen de una encuesta por muestreo, realizada en el verano de 1965, que abarcó 1640 hombres de 21 a 60 años de edad, residentes en el área metropolitana de Monterrey. ${ }^{9}$ Se diseñó una muestra estratificada por conglomerados, en dos etapas, en la que se dio mayor ponderación a la población masculina entre los 41 y los 60 años y a aquellos cuyos ingresos fuesen más elevados. De esta muestra ponderada, es posible pasar a una representativa al expandir las categorías subrepresentadas; sin embargo, salvo aclaración en este artículo, la información se refiere a la muestra real y no a la representativa. Los datos se obtuvieron de los entrevistados tanto por medio de preguntas específicas como por un cuestionario sobre la historia vital en el cual se registró la historia vital completa del entrevistado en temas como: educación, trabajo y todos los cambios de residencia cuya duración fuera de seis meses o más. ${ }^{10}$

9 Para mayor detalle sobre la encuesta y sus primeros resultados, véase Jorge Balan, Harley L. Browning y Elizabeth Jelin de Balan, comps., Movitidad social, migración y fecundidad en Monterrey metropolitano, Monterrey, Centro de Investigaciones Económicas, Facultad de Economía, Universidad de Nuevo León, 1967.

10 Una amplia discusión sobre el uso de la información de la historia vital aparece en Jorge Balán, Harley L. Browning y Elizabeth Jelin de Balán. "A Computerized Approach to the Processing and Analysis of Life Histories Obtained by 


\section{Resultados}

El cuadro 1 presenta las seis categorías de grupos migratorios tanto en la muestra real como en la representativa. Se verá que las diferencias entre las dos no son muy grandes pero que coinciden en cuanto a su sentido: los migrantes, cuya edad es mayor, están sobrerepresentados en la muestra real. Se incluye además en este cuadro un grupo que no se ha mencionado antes: el de los migrantes por adopción. Existen personas nacidas en Monterrey pero que se for-

\section{Cuadro 1}

MONTERREY : CLASIFICACIÓN MIGRATORIA DE LA POBLACIÓN MASCULINA DE 21 a 60 AÑOS DE EDAD. MUESTRA REAL Y REPRESENTATIVA

\begin{tabular}{ccc} 
Clasificación migratoria & $\frac{\text { Muestra (porciento) }}{\text { representativa real }}$ la muestra real en \\
\hline
\end{tabular}

Migrantes por nacimiento

(nacidos fuera de Monterrey y distinta comunidad de origen)

54

Años de residencia en Monterrey

A. menos de 10 años

B. de 10 a 19 años

C. más de 20 años

Migrantes por adopción

(nacidos en Monterrey y distinta comunidad de origen)

D. Nativos por adopción (nacidos fuera de Monterrey pero la comunidad de origen es Monterrey)

15

20

20

13

1

1 16

18

20

17

304

328

275

907

Nativos por nacimiento

(nacidos en Monterrey y la comunidad de origen es Monterrey)

E. Primera generación (niguno de sus padres nació en Monterrey)

F. Segunda generación

(uno o ambos padres nacieron en Monterrey)

30

29

489

17

15

254

Sin información

TOTAL

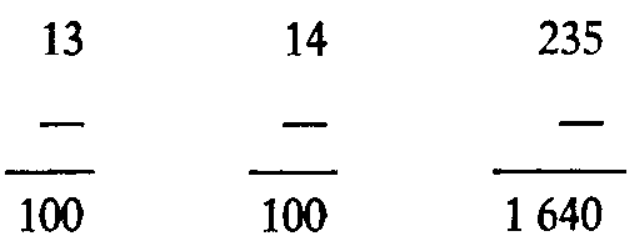

Nota: Acerca de la muestra real y representativa, véase el texto.

Sample Survey", Population Research Center, The University of Texas at Austin (mimeografiado). 
maron en otro lugar, y que estaban presentes de nuevo en Monterrey en el momento de la encuesta. Los 16 casos de este género comprendidos en la muestra real fueron tan pocos, para justificar su análisis, que se decidió no tenerlos en cuenta en lo que sigue.

La característica de mayor interés en el cuadro 1 es la proporción de la población masculina de Monterrey, entre los 21 y los 60 años, que se clasifica como migrante (con referencia en este caso a la muestra representativa). La proporción dependerá de la definición de migrante que se adopte. Si se utiliza el criterio de lugar de nacimiento, cerca de 7 de cada diez $(69 \%)$ de los individuos entre los 21 y los 60 años quedan en la categoría de migrantes. Aun excluyendo los nativos por adopción, más de la mitad (54\%) de la muestra tiene como lugar de origen una comunidad distinta a la de Monterrey. Apenas uno de cada siete adultos $(13 \%)$ es nativo de segunda generación. Debe recalcarse que este criterio de clasificación de migrante no es rígido, puesto que sólo requiere que uno de los padres haya nacido en Monterrey.

Estas cifras no debieran extrañar, en vista del rapidísimo crecimiento de Monterrey durante los últimós decenios a una tasa capaz de duplicar la población cada once años. Sin embargo, contribuyen a subrayar el hecho de que la gran mayoría de la población adulta en Monterrey ha llegado a la ciudad en época relativamente reciente $y$, por lo tanto, no ha tenido tiempo suficiente para arraigarse en la comunidad.

El cuadro 2 contiene la distribución porcentual de las características educación, ocupación, ingreso y vivienda, en cada grupo migratorio, lo que permite retomar la pregunta previamente establecida: ¿las categorías de grupos migratorios clasificadas por la duración de su exposición al medio ambiente urbano producen una progresión ordenada de cada uno de los cuatro indicadores del status socioeconómico?

En México, como en la mayoría de los países en desarrollo, la educación tal vez sea el mejor indicador para iniciar el análisis de este cuadro, sobre todo por el papel tan importante que desempeña en la localización ulterior de los individuos en la jerarquía ocupacional. A pesar de los esfuerzos decididos que se han hecho para mejorar el sistema educativo, existe aún en México una gran cantidad de analfabetos. Aun en Monterrey, centro metropolitano, localizado en una de las regiones más avanzadas del país, se encontró que una de cada siete personas, en la muestra representativa, se declaró incapaz de leer y escribir. La mayor parte de este grupo, como era de esperar, provenía de áreas rurales o de pequeñas poblaciones en donde no había escuelas o éstas eran insuficientes o inadecuadas. Aun cuando en el medio ambiente rural existan escuelas, los incentivos para asistir a la escuela formal son generalmente débiles.

Este punto se aprecia mejor al observar los datos relativos a personas que no han tenido educación formal. En los grupos $A$ y $B$, el $20 \%$ no concluyó un sólo año, mientras que en los grupos $E$ y $F$ sólo el $2 \%$ lo ha hecho. Los grupos $C$ y $D$ quedan en una situación intermedia. Los nativos por adopción ( grupo $D$ ) constituyen un grupo muy interesante porque no obstante haber estado expuestos al medio am- 
biente de Monterrey durante la mayor parte de sus años de formación, acusan una proporción mucho más elevada $(8 \%)$ de personas sin escolaridad que los nativos por nacimiento. Algunos llegaron a la ciudad entre los 8 y los 10 años, edad en que la mayoría de los niños ha iniciado ya su período escolar. En otros casos, es probable que ni el entrevistado ni sus padres estuvieran convencidos del valor de la educación, aun cuando pudieran tener acceso a ella. Adviértase también que mientras la primera generación de nativos no difiere de la segunda en cuanto a falta de escolaridad, aquélla está más propensa que ésta a desertar de la escuela antes de terminar la educación primaria, y que la segunda generación tiende más a completar su educación universitaria.

Al considerar el nivel ocupacional de las seis categorías de grupos migratorios conviene tener presente el grado de industrialización de Monterrey. El $82 \%$ de los migrantes con período corto de exposición (grupo $A$ ) son trabajadores manuales, y aun en el otro extremo el $54 \%$ de los nativos de segunda generación desempeñan trabajos manuales. ${ }^{11}$ La distribución según la ocupación muestra que la mayor discontinuidad ocurre entre los grupos $B$ y $C$, en particular al analizar a los trabajadores no calificados, cuya proporción baja de $40 \%$ en el grupo $B$ a $24 \%$ en el $C$. Existe en general mayor diferencia entre la primera y la segunda generación de nativos en cuanto a nivel ocupacional que en cuanto a educación. Llama la atención en la distribución ocupacional la escasa variación que existe entre los grupos $C$, $D$ y $E$. En todos los otros cuatro niveles ocupacionales no existe diferencia mayor de tres puntos porcentuales entre los migrantes con período largo de exposición, los nativos por adopción y los nativos de primera generación.

La distribución según el ingreso es similar a la de la ocupación. Nuevamente, las principales diferencias ocurren entre los grupos $B$ y $C$, siendo menores entre $\operatorname{los} E$ y $F$. La similitud de los grupos $C, D$ y $E$ es menos notable que en el caso de la ocupación. No existe progresión regular de $C$ a $E$. Los migrantes con período largo de exposición cuentan con mayor proporción de personas que reciben $\$ 580$ y más $(27 \%)$ que los nativos de primera generación $(21 \%)$.

La vivienda presenta una de las progresiones más ordenadas de las cuatro variables socioeconómicas. Por ejemplo, la combinación de los puntos 1 y 2 representa un nivel muy bajo de vivienda, puesto que son viviendas que no cuentan con servicios sanitarios interiores ni exteriores. El rango va desde $41 \%$ entre los migrantes con período corto de exposición a $11 \%$ entre los nativos de segunda generación. Nuevamente, entre los grupos $C, D$ y $E$ se dan casos de progresión no ordenada.

Para resumir las características generales del cuadro 2, se puede concluir que existen diferencias marcadas entre las categorías de grupos migratorios, y que existe una progresión definida entre los extremos de los grupos $A$ y $F$. No obstante, la progresión no consiste en

11 Debe puntualizarse que la clasificación de no manuales en este estudio es estricta, de forma que los trabajadores de cuello blanco son "verdaderamente" trabajadores de cuello blanco y los "gerentes" no son ni vendedores ambulantes ni propietarios de puestos callejeros. 


\section{Cuadro 2}

MONTERREY: CaTEgoRía MigRatoRia, SEGÚN EDUCACIÓN, OCUPACIÓN, INGRESO Y VIVIENDA

(En porcientos)

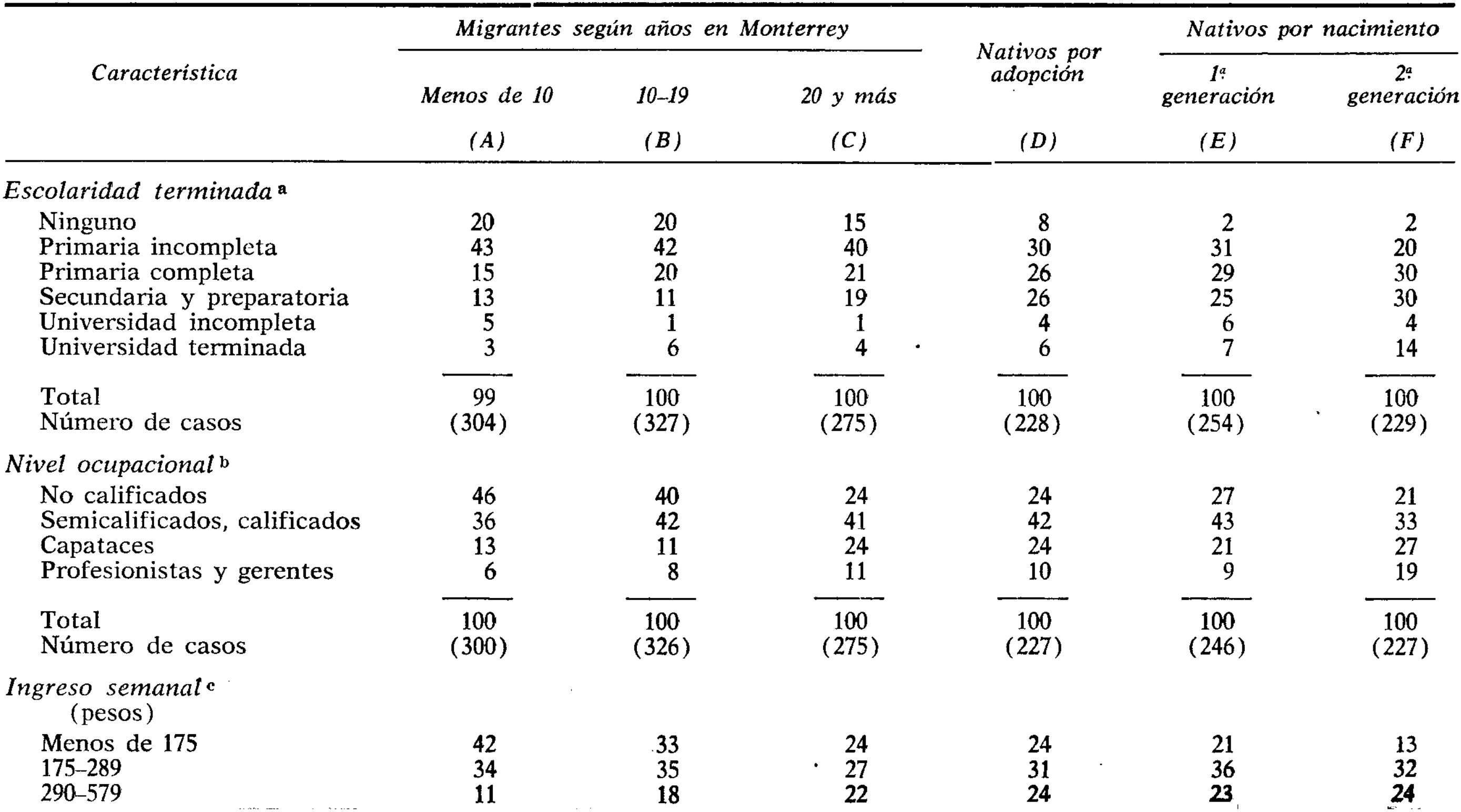




\begin{tabular}{|c|c|}
\hline $\begin{array}{l}9 \\
3\end{array}$ & $\begin{array}{r}10 \\
5\end{array}$ \\
\hline $\begin{array}{c}99 \\
(266)\end{array}$ & $\begin{array}{c}101 \\
(305)\end{array}$ \\
\hline
\end{tabular}

\begin{tabular}{r}
18 \\
9 \\
\hline 100 \\
$(245)$
\end{tabular}

\begin{tabular}{r}
12 \\
8 \\
\hline 99 \\
$(203)$
\end{tabular}

Total

Número de casos

$\begin{array}{r}7 \\ 21 \\ 24 \\ 14 \\ 18 \\ 8 \\ 3 \\ 4 \\ \hline 99\end{array}$

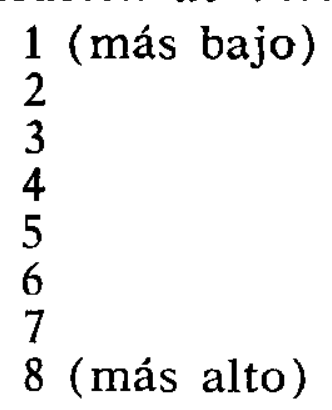

$\begin{array}{r}12 \\ 29 \\ 23 \\ 11 \\ 13 \\ 6 \\ 3 \\ 4 \\ \hline\end{array}$

Total

101

Número de casos

\begin{tabular}{r}
5 \\
15 \\
20 \\
19 \\
16 \\
10 \\
5 \\
10 \\
\hline 100 \\
$(274)$
\end{tabular}

\begin{tabular}{r}
7 \\
13 \\
23 \\
17 \\
18 \\
9 \\
6 \\
8 \\
\hline 101 \\
$(227)$
\end{tabular}

\begin{tabular}{r}
4 \\
12 \\
30 \\
14 \\
14 \\
10 \\
6 \\
9 \\
\hline 99 \\
$(251)$
\end{tabular}

a En el sistema educativo de México, la primaria es de seis años, la secundaria de tres, la preparatoria de 2 años y la universitaria cualquier número de años después de preparatoria.

b Esta clasificación se hizo con base en categorías ocupacionales detalladas, utilizando criterios estrictos. Véase nota del texto.

c Ingreso individual, no familiar. En 1965, el tipo de cambio era de 12.50 pesos por dólar. En la época de la encuesta el salario mínimo en Monterrey era de $\$ 145$ semanales. En las categorías migratorias los porcentajes de migrantes que están por debajo de este nivel fueron: $A, 19, B, 16 ; C, 10 ; D, 12 ; E, 7 ; F, 5$.

d Los siguientes ocho puntos utilizados en una escala de Guttman tienen un coeficiente de reproductibilidad de $0.97: 1)$ sin cocina ni baño; 2) facilidades de cocina (alguna clase de fuego, con alguna clase de techo y al menos una pared separándola del resto de la pieza o casa) ; 3) facilidades de baño (interior o exterior pero cercado por alguna clase de división y no compartido con otras familias); 4) al menos una pieza de la vivienda no se emplea como recámara; 5) menos de dos tercios de las piezas de la casa se emplean como recámaras; 6) menos de 1.1 personas por pieza; 7) al menos un sirviente de cualquier clase por período de horas, diario o sobre otra base, pero que no reside allí; y 8) al menos con un sirviente que vive allí mismo. 
diferencias graduadas y escalonadas, sino que su comportamiento se asemeja a una serie de mesetas, en las que los grupos de migrantes $A$ y $B$ forman la más baja, los $C, D$ y $E$ están a nivel intermedio (más bien irregular porque la progresión no siempre resultó como se esperaba) y el $F$ al nivel más elevado. ${ }^{12}$

El mayor acercamiento entre las categorías de grupos migratorios $C, D$ y $E$ fue una sorpresa. Considerando cualquiera de las dicotomías convencionales entre migrantes y nativos, habríase esperado que la separación principal ocurriese entre los grupos $C$ y $D$ o entre los grupos $D$ y $E$. $i$ Interviene otra variable que contribuye a disimular la dicotomía esperada? La variable en cuestión podría ser la edad. Como se verá en el cuadro 3 , dos de las categorías de grupos migratorios ( $B$ y $C$ ) tienen distribuciones por edad que difieren sustancialmente de las otras. Porque la duración de residencia no es independiente de la edad, esa característica podría esperarse. ${ }^{13}$ Se verá que para los migrantes con período largo de exposición no existen casos entre los 21 y los 30 años de edad. Esto es imposible por definición, a

\section{Cuadro $3^{\circ}$}

MonterRey: CATEgorfa Migratoria SEGÚN GRUPOS DE EDAD

(En porcientos)

\begin{tabular}{|c|c|c|c|c|c|c|}
\hline \multirow{2}{*}{$\begin{array}{l}\text { Grupos } \\
\text { de } \\
\text { edad }\end{array}$} & \multicolumn{3}{|c|}{$\begin{array}{c}\text { Migrantes segun años } \\
\text { en Monterrey }\end{array}$} & \multirow{2}{*}{$\begin{array}{c}\text { Nativos } \\
\text { por } \\
\text { adopcion } \\
(D)\end{array}$} & \multirow{2}{*}{$\frac{\text { Nativos por }}{1-}$} & \multirow{2}{*}{$\frac{\text { nacimiento }}{2 \text { a }} \begin{array}{c}\text { generacion } \\
(\mathrm{F})\end{array}$} \\
\hline & $\begin{array}{c}\text { menos de } 10 \\
\text { (A) }\end{array}$ & $\begin{array}{c}10-19 \\
(3)\end{array}$ & $\begin{array}{c}20 \text { y más } \\
\text { (c) }\end{array}$ & & & \\
\hline $\begin{array}{l}21-30 \\
31-40 \\
41-50 \\
51-60\end{array}$ & $\begin{array}{l}39 \\
20 \\
26 \\
16\end{array}$ & $\begin{array}{l}20 \\
23 \\
23 \\
23\end{array}$ & $\begin{array}{r}0 \\
19 \\
35 \\
46\end{array}$ & $\begin{array}{l}33 \\
20 \\
23 \\
19\end{array}$ & $\begin{array}{l}37 \\
25 \\
21 \\
16\end{array}$ & $\begin{array}{l}37 \\
19 \\
24 \\
20\end{array}$ \\
\hline Total & $\begin{array}{c}100 \\
(304)\end{array}$ & $\begin{array}{c}99 \\
(323)\end{array}$ & $\begin{array}{l}100 \\
(275)\end{array}$ & $\begin{array}{c}99 \\
(223)\end{array}$ & $\begin{array}{c}99 \\
(254)\end{array}$ & $\begin{array}{l}100 \\
(229)\end{array}$ \\
\hline Elad media & 35.7 & $41 \cdot 5$ & 50.3 & 36.9 & 36.4 & 37.2 \\
\hline
\end{tabular}

12 Una forma gruesa pero efectiva de demostrar este punto es en términos del "índice de aprovechamiento". Se construye sumando simplemente los porcentajes en las categorías más elevadas dentro de cada una de las cuatro variables y cada uno de los seis grupos. Así el grupo A tiene un $3 \%$ que terminó su preparación universitaria, $6 \%$ que son profesionistas y gerentes, $3 \%$ que están en la categoría más alta según el ingreso personal y $4 \%$ en la categoría más alta en relación con la vivienda, lo que hace un total de 16. Las cifras de los seis grupos son:

$$
\begin{array}{llllll}
A & \frac{B}{16} & \frac{C}{23} & \frac{D}{34} & \frac{E}{32} & \frac{F}{60}
\end{array}
$$

La separación mayor es entre los grupos $B$ y $C$ (11 puntos) y entre $E$ y $F$ (27 puntos). Entre cualquier otra combinación, la diferencia más amplia es de sólo 7 puntos.

13 Esto podría deberse en parte al hecho de que la selección de los entrevistados no se restringió a los jefes de hogar. Los entrevistados más jóvenes con altos ingresos y ocupación podrían haber estado residiendo con sus padres de status más bajo en viviendas más pobres. 
causa del requisito de 20 años o más de residencia en Monterrey. Cabe también destacar que el grupo migratorio $A$ muestra una distribución por edad similar a la de los grupos $D$ a $F$.

\section{Cuadro 4}

MONTERREY: VALORES PROMEDIO EN EDUCACIÓN, OCUPACIÓN, INGRESO $Y$ VIVIENDA POR CATEGORIA MIGRATORIA Y EDAD

\begin{tabular}{|c|c|c|c|c|c|c|c|c|c|c|c|}
\hline \multirow{2}{*}{$\begin{array}{l}\text { Carroterlotions } y \\
\text { grupos de edad }\end{array}$} & \multicolumn{11}{|c|}{ Categorfan Migratorias } \\
\hline & $A$ & & B & & c & & D & & $\mathbf{B}$ & & $\mathbf{F}$ \\
\hline \multicolumn{12}{|l|}{ Educrcion } \\
\hline $\begin{array}{l}21-30 \\
31=40 \\
41=50 \\
51=60\end{array}$ & $\begin{array}{l}6.05 \\
4.55 \\
3.76 \\
2.47\end{array}$ & & $\begin{array}{l}6.75 \\
4.97 \\
4.25 \\
2.78\end{array}$ & & $\begin{array}{l}- \\
5.60 \\
5.73 \\
4.26\end{array}$ & & $\begin{array}{l}6.62 \\
6.40 \\
6.67 \\
5.31\end{array}$ & & $\begin{array}{l}8.40 \\
6.51 \\
5.31 \\
5.82\end{array}$ & & $\begin{array}{l}9.45 \\
8.30 \\
7.08 \\
6.50\end{array}$ \\
\hline $\begin{array}{l}\text { Probabilidad, } \\
\text { eliminando el } \\
\text { electo de la edad b/ }\end{array}$ & & 0.66 & & .01 & & . 30 & & .02 & & .00 & \\
\hline Media & 4.59 & & 4.61 & & 5.01 & & 6.33 & & 6.36 & & 3.17 \\
\hline $\begin{array}{l}\text { Probabilidad, } \\
\text { sin eliminar el } \\
\text { efecto de la edad b/ } \\
\text { Mivel oouproional o/ }\end{array}$ & & 1.00 & & .24 & & .00 & & .17 & & .00 & \\
\hline $\begin{array}{l}21-30 \\
31-40 \\
41=50 \\
51-60\end{array}$ & $\begin{array}{l}1.26 \\
1.25 \\
1.32 \\
.91\end{array}$ & & $\begin{array}{r}1.70 \\
1.43 \\
1.41 \\
.81\end{array}$ & & $\begin{array}{l}1.65 \\
2.15 \\
1.83\end{array}$ & & $\begin{array}{l}1.58 \\
2.04 \\
2.07 \\
1.98\end{array}$ & & $\begin{array}{l}1.54 \\
1.82 \\
1.96 \\
1.85\end{array}$ & & $\begin{array}{l}2.14 \\
2.73 \\
2.40 \\
2.55\end{array}$ \\
\hline $\begin{array}{l}\text { Probabilidad olimi- } \\
\text { nando el efecto de } \\
\text { la odad b/ }\end{array}$ & & $\cdot 55$ & & .00 & & .94 & & .94 & & .00 & \\
\hline Media & 1.21 & & 1.33 & & 1.94 & & 1.87 & & 1.76 & & 2.40 \\
\hline $\begin{array}{l}\text { Probabilidad, sin } \\
\text { eliminar el efecto } \\
\text { do la edad b/ } \\
\text { Ingreso Semanal d/ }\end{array}$ & & .61 & & .00 & & .65 & & .50 & & .00 & \\
\hline $\begin{array}{l}21=30 \\
31=40 \\
41=50 \\
51=60\end{array}$ & $\begin{array}{l}2.25 \\
2.25 \\
2.81 \\
1.83\end{array}$ & & $\begin{array}{l}2.89 \\
2.96 \\
3.03 \\
1.72\end{array}$ & & $\begin{array}{l}3 . \\
3.23 \\
4.00 \\
3.50\end{array}$ & & $\begin{array}{l}2.55 \\
3.76 \\
3.78 \\
3.88\end{array}$ & & $\begin{array}{l}3.00 \\
3.53 \\
4.05 \\
3.16\end{array}$ & & $\begin{array}{l}3.40 \\
4.58 \\
4.43 \\
4.28\end{array}$ \\
\hline $\begin{array}{l}\text { Probabilidad, eli- } \\
\text { minando el efecto } \\
\text { de la edad b/ }\end{array}$ & & .15 & & .00 & & .71 & & .53 & & .03 & \\
\hline Media & 2.32 & & 2.69 & & 3.62 & & 3.34 & & 3.41 & & 4.07 \\
\hline $\begin{array}{l}\text { Probabilidad sin } \\
\text { eliminar el efecto } \\
\text { de la edad b/ }\end{array}$ & & .06 & & .00 & & .21 & & .74 & & .00 & nín) \\
\hline
\end{tabular}


Cuadro 4 (continuación)

\begin{tabular}{|c|c|c|c|c|c|c|c|c|c|c|c|}
\hline \multirow{2}{*}{$\begin{array}{c}\text { Caracteristicas y } \\
\text { grupos de edad }\end{array}$} & \multicolumn{11}{|c|}{ Categorlas Migratorias } \\
\hline & $A$ & & B & & c & . & D & & $\mathbf{E}$ & & $\mathbf{F}$ \\
\hline \multicolumn{12}{|l|}{ Vivienda e/ } \\
\hline $\begin{array}{l}21-30 \\
31=40 \\
41-50 \\
51-60\end{array}$ & $\begin{array}{l}2.30 \\
2.41 \\
2.33 \\
2.39\end{array}$ & & $\begin{array}{l}2.76 \\
2.79 \\
2.60 \\
2.55\end{array}$ & & $\begin{array}{l}-\overline{8} \\
2.84 \\
3.38 \\
2.87\end{array}$ & & $\begin{array}{l}2.74 \\
3.19 \\
3.18 \\
3.98\end{array}$ & & $\begin{array}{l}3.30 \\
3.17 \\
3.00 \\
3.41\end{array}$ & & $\begin{array}{l}3.65 \\
3.49 \\
4.00 \\
3.65\end{array}$ \\
\hline $\begin{array}{l}\text { Probabilidad, eli- } \\
\text { minando el efecto } \\
\text { de la edad b/ }\end{array}$ & & .28 & & .00 & & .22 & & .25 & & .03 & \\
\hline Media & 2.35 & & 2.68 & & 3.23 & & 3.17 & & 3.21 & & 3.71 \\
\hline $\begin{array}{l}\text { Prokabilidad sin } \\
\text { eliminar el efec- } \\
\text { to de la edad b/ }\end{array}$ & & .03 & & .00 & & .73 & & .79 & & .00 & \\
\hline
\end{tabular}

Número de años de escolaridad terminados.

b Hipótesis nula de que la diferencia entre los grupos migratorios adyacentes se debe al azar. Por ejemplo, la probabilidad, bajo la hipótesis nula, de que $A=B$ en educación es $p=0.66$. Formalmente, esto significa que la probabilidad de que los valores obtenidos del modelo, dejando variar libremente las medias de las clases de migrantes dentro de cada grupo, pudieran haber sido obtenidos muestreando al azar una población en que los valores en los grupos $A$ y $B$ sean iguales en cada nivel a 0.66 , si es que se cumplen los supuestos de la distribución $F$.

c Escala de seis puntos, de 0 (trabajadores no calificados) a 6 (gerentes y profesionistas).

d Escala de nueve puntos, con intervalos desiguales, 0 a 8.

e Escala de ocho puntos, 0 a 7 . Véase el cuadro 2 .

En consecuencia, en el cuadro 4 se introduce la edad. Se divide el grupo total en cuatro grupos decenales y para cada uno de estos grupos se obtiene una media y luego la media para el total de los cuatro grupos, considerando las cuatro variables socioeconómicas. Se incluye además en el cuadro la probabilidad de que las diferencias entre los grupos adyacentes sean producto del azar, controlando o no la variable edad.

Analizando primero las cifras medias por grupos de edad es evidente que las diferencias entre los grupos subsisten y que la progresión existe para todos los grupos de edad. La distancia entre los grupos extremos $A$ y $F$ es muy grande con respecto a la media total. Sin embargo, las diferencias no se amplían regularmente conforme se aumenta la edad. Los grupos de edad, al igual que la media total, para cada una de las variables socioeconómicas, presentan grandes separaciones entre los grupos $B$ y $C$ y los grupos $E$ y $F$. Los grupos $C, D$ y $E$ presentan nuevamente poca variación y la que existe no presenta progresión congruente.

Aunque nuestro principal interés en el cuadro 4 es la diferencia entre las categorías de grupos migratorios, es importante, sin embargo, considerar dentro de cada grupo las variaciones según la edad. La educación presenta un comportamiento fijo, con muy pocas ex- 
cepciones. Existe considerable relación inversa entre los aumentos de edad y el número de años de escolaridad cumplidos. De todos los grupos, los migrantes por adopción (grupo $D$ ) inexplicablemente presentan las diferencias más pequeñas en cuanto a logros educacionales por edad. El mejoramientó más notable es el de los grupos $A$ y $B$, en los que existe una diferencia de casi cuatro años entre los grupos de edad más jóvenes y más viejos. En el caso de México estas cifras eran previsibles, porque las generaciones recientes han contado con más amplias facilidades educativas, siendo mayor el mejoramiento en las áreas rurales, de donde provienen muchos de los migrantes.

Lo que más llama la atención en la distribución ocupacional es la diferencia tan considerable en el número de años de escolaridad terminados entre los grupos migratorios $A$ y $B$, por un lado, y el $C$, por otro. Mientras que para los de las edades 31-40 la diferencia entre el grupo $A$ y el $C$ es de 1.05, años, aumenta a 1.97 años en las edades 41-50, y es de 1.79 años en las edades 51-60. Lo anterior sugiere que el grupo $C$ de migrantes, que contaba con un período de residencia mayor en Monterrey, fue más selectivo en términos de educación de lo que lo fueron los grupos $A$ y $B$.

El nivel ocupacional muestra un comportamiento muy diferente. Ya que 3.00 representa la división entre manuales y no manuales, deberá puntualizarse que todas las medias caen dentro de la categoría de manuales (los nativos de segunda generación con edades de 31 a 40 años tienen la calificación más alta, 2.73). La relación entre el nivel ocupacional y la edad es claramente curvilínea, puesto que las edades 51-60 no alcanzan la calificación más elevada en ninguno de los grupos. Los grupos $A$ y $B$ presentan descensos notorios entre las dos últimas clases de edad. En un país como México, entre los obreros no calificados casi no existen carreras ocupacionales ordenadas, $y$ si una persona sólo ofrece su fuerza manual en el mercado de trabajo, la posibilidad de encontrar empleo disminuye conforme aumenta la edad; sobre todo si se trata de una persona que ha emigrado recientemente. Una vez más el grupo $C$ difiere de los otros dos grupos de migrantes, y se encuentra más cercano a $D, E$ y $F$, entre los cuales existe apenas leve descenso, o no se registra ninguno.

El mismo tipo de relación subsiste con relación al ingreso. En cuatro de los seis casos la calificación más alta se encuentra en las edades 41-50. Como era de esperar, los grupos $A$ y $B$ acusan un descenso significativo en la última clase de edad.

Finalmente, respecto a vivienda se encontró que el efecto de la edad tiene menor influencia en su comportamiento del que tuvo en las otras variables. Por otro lado, en vivienda existen diferencias significativas entre los grupos según la duración de residencia en contacto con Monterrey, pero dentro de cada uno de los grupos la edad tiene escaso efecto. ${ }^{14}$

El cuadro 4, como se expresó anteriormente, incluye la probabilidad, controlando o no la edad, de que las diferencias entre los grupos adyacentes de las categorías de grupos migratorios se produzcan al

14 Deberá puntualizarse que las diferencias entre los grupos $E$ y $F$ no son de una generación como se desprende de los títulos, sino de dos o más para la segunda generación de nativos. 
azar. Aquí confirmamos lo que anteriormente se había sugerido. Sin excepción, cuando se controla la edad, la probabilidad de que las diferencias entre los grupos $B$ y $C$ y los grupos $E$ y $F$ se deban al azar es muy baja. En el caso de la vivienda, no es tan clara la falta de diferencias en el comportamiento. Pero los grupos $A$ y $B$ son similares en cuanto a logros educacionales y se confirma el acercamiento ya señalado antes entre $C, D$ y $E$ en cuanto a ocupación e ingreso.

Otra forma de presentar esta información aparece en el cuadro 5, donde se prueba la hipótesis nula para diferentes combinaciones de las categorías de grupos migratorios, así como el número de veces que se rechaza o se acepta con relación a las cuatro variables socioeconómicas, con y sin control de edad.

En la primera sección del cuadro se verifica la hipótesis nula para los grupos adyacentes y se confirma la configuración observada en el cuadro 4. Para cada una de las cuatro variables socioeconómicas, cuando se controla la edad, las hipótesis $B=C$ y $E=F$ se rechazan. Por el contrario, las hipótesis $A=B$ y $C=D$ no se rechazan en ningún caso y $D=E$ se rechaza en tres de los cuatro casos.

La segunda sección presenta la hipótesis nula para grupos no adyacentes, $A=F$ y $B=E$; en cada caso la hipótesis nula de que las diferencias se deban al azar se rechaza; y cuando se controla la edad,

\section{Cuadro 5}

Pruebas de LAS HIPÓtesis NULAS SOBRE LAS RELACIONES ENTRE LOS GRUPOS MIGRATORIOS, CONTROLANDO O NO LA VARIABLE EDAD

\begin{tabular}{|c|c|c|c|c|c|c|c|c|}
\hline \multirow{3}{*}{$\begin{array}{l}\text { Hipotesis } \\
\text { nulas b/ }\end{array}$} & \multicolumn{4}{|c|}{$\begin{array}{l}\text { Controlando la } \\
\text { vartable edad }\end{array}$} & \multicolumn{4}{|c|}{$\begin{array}{c}\text { No controlanio la } \\
\text { variable edsd }\end{array}$} \\
\hline & \multicolumn{2}{|c|}{$\begin{array}{l}\text { Numero de va } \\
\text { riables para } \\
\text { que las hipo } \\
\text { tesis a }\end{array}$} & \multicolumn{2}{|c|}{$\begin{array}{c}\text { Rechazadas oon } \\
\text { diferencias } \\
\text { significativas } \\
\text { al nivel de }\end{array}$} & \multicolumn{2}{|c|}{$\begin{array}{l}\text { Numero de va } \\
\text { riables para } \\
\text { que las hipo } \\
\text { tesis a }\end{array}$} & \multicolumn{2}{|c|}{$\begin{array}{c}\text { Rechazadas con } \\
\text { diferencias } \\
\text { significativa } \\
\text { al nivel de }\end{array}$} \\
\hline & $\begin{array}{l}\text { No ge } \\
\text { neoha } \\
\text { oen }\end{array}$ & $\begin{array}{l}\text { Se re } \\
\text { cha } \\
\text { cen }\end{array}$ & .01 & .05 & $\begin{array}{l}\text { No se } \\
\text { recha } \\
\text { cen }\end{array}$ & $\begin{array}{l}\text { Se re } \\
\text { cha } \\
\text { cen }\end{array}$ & .01 & .05 \\
\hline$A=B$ & 4 & 0 & - & - & 4 & 0 & - & - \\
\hline$B=C$ & 0 & 4 & 3 & 1 & $i$ & 3 & 3 & - \\
\hline$C=D$ & 4 & 0 & - & - & 3 & 1 & 1 & - \\
\hline$D=E$ & 3 & 1 & - & 1 & 4 & 0 & - & - \\
\hline$F=F$ & 0 & 4 & 2 & 2 & 0 & 4 & 4 & - \\
\hline$A=F$ & 0 & 4 & 4 & - & 0 & 4 & 4 & - \\
\hline$B=E$ & 0 & 4 & 3 & 1 & 0 & 4 & 4 & - \\
\hline$A=B=C$ & 0 & 4 & 4 & - & 1 & 3 & 3 & - \\
\hline$B=C=D$ & 0 & 4 & 4 & - & 0 & 4 & 4 & - \\
\hline$C=D=E$ & 3 & 1 & - & 1 & 3 & $i$ & 1 & 一 \\
\hline$D=E=F$ & 0 & 4 & 3 & 1 & 0 & 4 & 4 & - \\
\hline$A B C=D E F$ & 0 & 4 & 4 & - & 0 & 4 & 4 & - \\
\hline$A B C D=E F$ & 0 & 4 & 4 & - & 0 & 4 & 4 & - \\
\hline$A B=C D E F$ & 0 & 4 & 4 & - & 0 & 4 & 4 & - \\
\hline
\end{tabular}

a Las cuatro variables son educación, ingreso, ocupación y vivienda.

b Las letras se refieren a las seis categorías migratorias. Para su explicación, véase el cuadro 1. 
siete de cada ocho casos están al nivel de .01. En otras palabras, la noción de una progresión se sostiene hasta el punto en que existan diferencias significativas entre los grupos en los extremos de la clasificación.

Cuando se verifica la hipótesis nula de que tres de los grupos adyacentes no presentan diferencias, de las cuatro posibles combinaciones sólo en el grupo del centro $C=D=E$ se acepta con tres de las cuatro variables (exceptuándose la educación).

Por último, en la cuarta sección los seis grupos se dicotomizan en tres combinaciones. La primera, $A B C=D E F$, corresponde a la dicotomía entre nativos y migrantes; cuando son nativos por adopción (D) se colocan en la categoría de nativos. La segunda, $A B C D=E F$ corresponde a la forma en que se presenta la información censal, según lugar de nacimiento. La tercera posibilidad $A B=C D E F$ no tiene ningún significado independiente excepto la duración del período de residencia en la ciudad. La hipótesis nula de las tres dicotomías se rechaza uniformemente en todos los casos al nivel de .01.

\section{COMENTARIOS}

¿Qué significan estos resultados acerca de Monterrey y, en particular, cómo pueden relacionarse con los problemas que planteamos al principio de este estudio? Con respecto al primer problema planteado (véase la p. 185), la respuesta es que en general existe progresión general, con grandes diferencias entre los grupos extremos $(A$ y $F$; $B$ y $E$ ), pero que la progresión no es siempre ordenada. La inconsistencia se encuentra en los grupos centrales $(C, D$ y $E$ ) de modo que los migrantes con período largo de exposición $(C)$ tendrán en algunos casos una clasificación más alta que los nativos de primera generación $(E)$. No existe tampoco un espaciamiento regular entre los seis grupos, aun cuando su clasificación se ordene desde $A$ a $F$. Pero tampoco existe una sola separación que nos permitiera establecer la dicotomización en forma "natural" de los dos grupos, o sea en migrantes y nativos, sino que el comportamiento presenta dos separaciones importantes y produce una tricotomía en vez de una dicotomía. El comportamiento general se ilustra en la gráfica 1. En vez de una progresión ordenada de los seis grupos, aparecen tres "mesetas" $A B-C D E-F$. Obviamente, cada una de las variables socioeconómicas difiere algo en su ubicación, pero en ningún caso se presenta algo semejante a una dicotomía.

Quizás sea más importante -y más extraño- el hecho de que las dos principales separaciones (entre los grupos $B$ y $C, E$ y $F$ ) no son las discontinuidades usuales que se presentan cuando se clasifica la población en migrantes y nativos (entre $D$ y $E$ o entre $C$ y $D$ ). Sin embargo, esto no significa que dicotomizar en lugares "equivocados" produce diferencias sin sentido entre migrantes y nativos. Como en verdad existe una progresión y como también hay diferencias muy importantes entre los grupos extremos, cualquier separación por la que uno dicotomice en el área central (como se indica en la última sección del cuadro 5) producirá diferencias estadísticamente significativas y teóricamente válidas. 


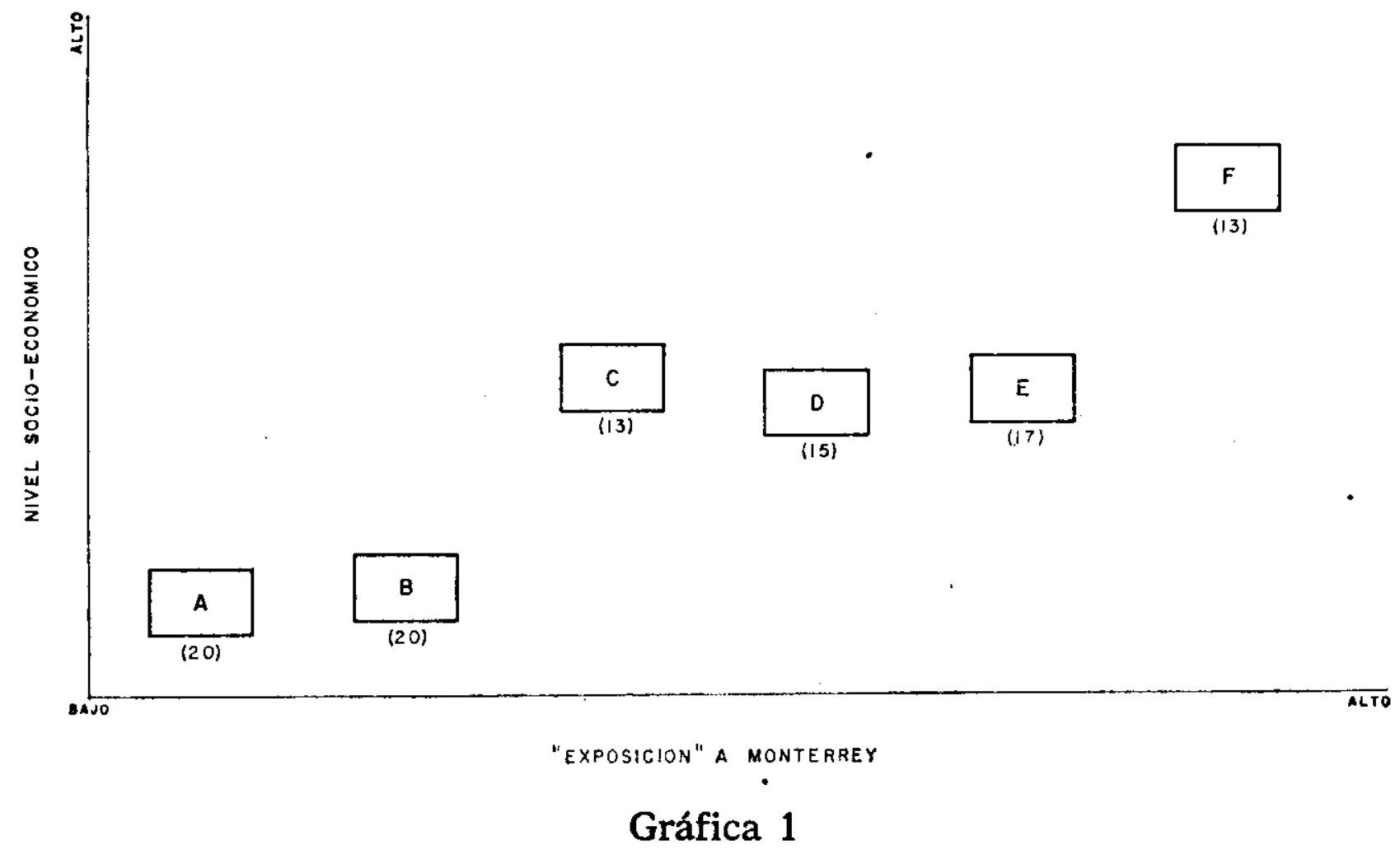

REPRESENTACIÓN GRAFICA DE LAS CATEGORfAS MIGRATORIAS *

* Los datos entre paréntesis representan la proporción de los grupos en la muestra representativa total con respecto a hombres de 21 a 60 años.

Como el problema de dónde dicotomizar una distribución es en el fondo una decisión técnica, hecha con propósitos heurísticos, examinemos ahora el problema más importante que consiste en señalar la existencia de las separaciones inesperadas entre los grupos $B$ y $C, E$ y $F$. A simple vista parece fácil señalar la gran distancia entre los nativos de primera y segunda generaciones estableciendo una analogía entre ellos y los migrantes de la primera y segunda generaciones. La mayoría, si no todos los estudios, ha demostrado que la segunda generación aventaja claramente a la primera con respecto a las características socioeconómicas utilizadas en esta investigación. Pero las analogías son engañosas. A diferencia de los emigrantes de la primera generación nacidos y a menudo criados en otros países, y que generalmente sufren la desventaja adicional de tener que aprender otro idioma siendo ya adultos, los nativos de primera generación en Monterrey no cargan con esta desventaja. Ellos nacen y crecen en Monterrey y no tienen sino una lengua.

La edad puede ser un factor importante. Los nativos de la primera y segunda generaciones registran diferencias muy marcadas entre las cuatro variables socioeconómicas, y mientras es cierto que los nativos de la segunda generación son algo mayores, queda aún una diferencia importante después de eliminar el efecto de la edad.

El que una persona haya nacido y crecido en una zona urbana no es en sí mismo decisivo para el grupo $E$, puesto que en algunos casos queda más próxima a los migrantes con período largo de exposición (C) de lo que estaría respecto a los nativos de segunda generación (excepto en educación). Los antecedentes familiares parecen ser decisivos, porque el criterio que se utiliza para distinguir los grupos $E$ 
y $F$ es que uno de los padres haya nacido en Monterrey y porque, además, los padres de la segunda generación de nativos tienen niveles educacionales y ocupacionales más altos que los de la primera generación.

Por ejemplo, el $22 \%$ de los padres del grupo $E$ no tenía años de escolaridad, comparado con un $8 \%$ del grupo $F$. Para las madres, fue 26 y $15 \%$, respectivamente. Considerando el nivel ocupacional de los padres a una edad más próxima a la de sus hijos en el momento de la encuesta, el $26 \%$ de los padres del grupo $E$ estaba en el más bajo, esto es, el nivel de no calificados, mientras que el de los padres de los del grupo $F$ era $15 \%$. Esto sugiere que la absorción de los migrantes a la estructura socioeconómica de la ciudad es un proceso lento que requiere al menos dos generaciones.

En cierto modo, tanto la separación entre los grupos $B$ y $C$ como el acercamiento de los grupos $C, D$ y $E$ son tan complejos y difíciles de explicar como la diferencia existente entre los grupos $E$ y $F$. Como la discontinuidad y el agrupamiento se relacionan entre sí, las consideraremos conjuntamente, empezando por el último. La similitud general de los nativos por adopción $(D)$ y los nativos de la primera generación $(E)$ no debería extrañar puesto que los antecedentes de los padres no difieren mucho. El que un individuo se clasifique o no como $D$ o $E$ es con frecuencia cuestión de tiempo, ya que depende de si su nacimiento se produjo antes o después de la llegada de sus padres a Monterrey.

El problema de fondo es el de explicar los migrantes con período largo de exposición $(C)$, que están más separados de los otros migrantes (grupo $A$ y $B$ ) y más próximos a los grupos $D$ y $E$ de lo que podría esperarse. La similitud general entre los migrantes con período largo

\section{Cuadro 6}

VALORES PROMEDIOS SEGÚN EDUCACIÓN, OCUPACIÓN, INGRESO Y VTVIENDA PARA DOS CATEGORIAS MIGRATORIAS, CADA UNA CON 20 O MÁS AÑOS

EN MONTERREY Y 40 o Más AÑOS DE EDAD

\begin{tabular}{lcc}
\hline Caracteristica & $\begin{array}{c}\text { Nativos } \\
\text { por } \\
\text { adopción } \\
\text { (C) }\end{array}$ & $\begin{array}{c}\text { Migrantes con } \\
\text { periodo largo } \\
\text { de exposición } \\
\text { (D) }\end{array}$ \\
\hline Educación a $^{\text {a }}$ & 4.1 & 3.6 \\
Ocupación b $^{\text {b }}$ & $(53)$ & $(115)$ \\
Ingreso $^{c}$ & 1.8 & 2.1 \\
Vivienda $^{d}$ & $(53)$ & $(115)$ \\
& 4.6 & 4.8 \\
& $(45)$ & $(106)$ \\
\hline
\end{tabular}

a Escala de nueve puntos, desde 0 años de escolaridad (1) hasta universidad terminada (9).

b Escala de siete puntos, desde trabajador no calificado (1) a gerentes y profesionistas (7).

c Escala de nueve puntos, con intervalos desiguales.

d Véase cuadro 2, nota $d$. 
de exposición y los nativos por adopción no se ajusta a los lineamientos del análisis anterior que daba mucha importancia al lugar donde transcurrieron los años de formación (edades 5-15) y que servía para justificar la creación del grupo $D$. .

La relación entre los grupos $C$ y $D$ se examina en el cuadro 6, donde el período de residencia en Monterrey en la edad adulta se mantiene constante. Sostuvimos antes que el acceso a los mejores servicios educativos que ofrece un centro urbano grande contribuía a que los nativos por adopción se clasificasen en rango más elevado que cualquier otro grupo de migrantes porque la educación es decisiva en la obtención de mejores ingresos y ocupaciones. El cuadro 6 confirma las diferencias en logros educativos. No se registran, sin embargo, diferencias significativas en los niveles de ocupación e ingreso alcanzados. ¿Cómo es posible que los migrantes con período largo de exposición compitan en condiciones iguales con los nativos por adopción a pesar de su desventaja educacional?

La respuesta quizá se halle en el concepto de "cristalización" de la estructura ocupacional.15 Básicamente, cuanto más cristalizada sea una estructura de ocupación, más específicos y formales serán los criterios que gobiernen la posibilidad de alcanzar efectivamente una posición ocupacional. Con frecuencia, aunque no siempre, estos criterios se establecen en términos de adiestramiento y educación formales. Puesto que los hombres en el grupo $C$ de migrantes han pasado por lo menos 20 años en Monterrey, significa que ellos llegaron antes de 1945, época en que el desarrollo económico de México se encontraba en etapa de expansión como resultado de la demanda externa creada por la segunda Guerra Mundial. Estos hombres fueron absorbidos en primera instancia a pesar de su bajo nivel educativo. Entre 1945 y 1965, el período que abarca la encuesta, la estructura por ocupaciones ha llegado a ser más rígida, aunque no se puede ser preciso debido a las limitaciones de la información. ${ }^{16} \mathrm{Si}$ esta tendencia persiste, quiere decir que los grupos $C$ y $D$ se diferencian más a medida que los requisitos educativos y otras calificaciones necesarias para ocupar posiciones se vuelven más estrictos.

Queda aún por considerar la gran distancia entre los grupos migratorios $B$ y $C$; aquellos con 10 a 19 y 20 años y más en Monterrey, respectivamente. Al elaborar una interpretación razonable no habría problema si la separación se presentase entre los grupos $A$ y $B$, pues podría sostenerse que los migrantes con período corto de exposición (menos de 10 años) y cuya llegada ha sido más reciente incluían en-

15 Véase Jorge Balán, Structural Analysis of Socio-Economic Differences between Migrants and Natives in Latin American Cities, documento presentado a la Conferencia sobre Movilidad Humana y Cambio Social en América Latina y Africa, Universidad de Florida, Gainesville, noviembre 29-diciembre 2, 1967; también Otis Dudley Duncan y Leo F. Schnose, "Cultural Behavioral and Ecological Perspectives in the Study of Social Organization", American Journat of Sociology, LXIV, septiembre de 1959 , p. 140.

16 El $53 \%$ de la muestra representativa total estaba ocupado en empresas con 50 a más trabajadores. La gran mayoría de las empresas de este tamaño tiene contratos colectivos. Estos contratos en forma creciente especifican en detalle la posición en la ocupación y quién puede ocuparla. En algunos contratos se ha introducido la condición de que los individuos tienen que haber completado la educación primaria (seis años) antes de poder ocupar una posición manual. 
tre ellos un número considerable que acusaba grandes dificultades para encontrar un lugar en la estructura económica y social de Monterrey. Es de suponer también que una gran proporción del grupo $A$, mayor que la de los otros dos grupos migratorios, sean migrantes frustrados, que por una razón u otra son incapaces de adaptarse a la vida urbana y que tarde o temprano, dentro de un intervalo de 10 años, saldrán de Monterrey. El grupo $B$ de migrantes no contaría, seguramente, con tantos fracasos migratorios como el grupo $A$, porque todos los hombres tienen al menos 10 años en Monterrey. $Y$ sin duda un período de 10 a 20 años proporcionaría suficiente tiempo para adaptarse al medio ambiente metropolitano.

¿Por qué, entonces, el grupo $B$ se presenta tan desfavorable en cuanto a logros cuando se compara con el grupo $C$ ? Por ahora sólo es posible hacer una interpretación provisional,17 pero una investigación preliminar sugiere que los hombres que forman el grupo $C$ son un grupo más selecto de la población donde se origina que aquellos que forman los grupos $B$ o $A$. Este punto fue tocado ya cuando se habló de los logros educativos por grupos de edad (cuadro 4). Se vio que el grupo $C$, al menos en las edades 41 y más tenía niveles educativos más elevados que su contraparte en los grupos $A$ y $B$. Aunque aquí no se pueden presentar los datos, se encontró también que los integrantes del grupo $C$ tenían padres cuyos niveles educativos eran más altos que los de los otros grupos $A$ y $B$. Los miembros del grupo $C$ tendieron a alejarse de su comunidad de origen a edad más temprana, muchos eran solteros y en promedio casaron a una edad mayor que los de los grupos $A$ y $B$. En resumen, los primeros migrantes parecen ser más selectos bajo diversos criterios. Fueron más "pioneros" que los migrantes anteriores "en masa". Podríamos decir que estaban preparados y en situación de aprovechar la menor rigidez de la estructura ocupacional.

\section{CONCLUSIONES}

Si se preguntara si es realmente significativo dicotomizar nativos y migrantes como se hace comúnmente, la respuesta tendría que ser ambigua. Los resultados de este documento respaldan en forma general la existencia del continuum nativo-migrante al verificarlo con importantes indicadores socioeconómicos. Buena parte de la discusión se ha dedicado a tratar de entender el comportamiento inesperado que se presentó. Para aquellos investigadores cuya única alternativa es utilizar la clasificación de tipo censal entre nativos y migrantes hemos demostrado que se puede dicotomizar en un lugar "equivocado" y aun obtener resultados útiles e interpretables dada la existencia del continuum. Para otros investigadores que deban reunir su propia información, un diseño de investigación con la dicotomía nativo-migrante será suficiente para su análisis siempre que tengan un interés limitado en la categoría migratoria. Es conveniente, sin embargo, hacer la separación de acuerdo con la comunidad en la que el individuo pasó sus años formativos en vez del lugar de nacimiento.

17 Está en elaboración un análisis detallado de migrantes según su situación social y lugar de origen. 
No obstante, al establecer el diseño de la mayor parte de las investigaciones de campo, que abarcan desde la fecundidad hasta las relativas a actitudes políticas, nos parece recomendable que no se tome como base la dicotomía nativo-migrante, çomo quiera que ésta se haga, porque tiende a ocultar diferencias importantes que serían de gran utilidad en la interpretación del tópico central de la investigación. Hemos tratado de demostrar que la categoría migratoria es compleja y que posee características variadas y sutiles. No existe, por lo tanto, un número fijo de categorías migratorias, sino que más bien dependerá de las necesidades y recursos de los programas de investigación.

Una de las conclusiones principales que se desprende de nuestro estudio es la naturaleza "situacional" de las diferencias entre nativos y migrantes. Hemos introducido un número de factores que tienen y ejercen un impacto importante en la actuación relativa de los nativos y los migrantes, entre otros el nivel y el ritmo del desarrollo económico del país, la distribución urbano-rural, y la proporción dada de nativos y migrantes en una localidad. Utilizando este último factor como ilustración considérese el hecho de que en una ciudad como Monterrey, que crece rápidamente, y en un país como México, cuatro de cada cinco adultos están a dos generaciones de un medio ambiente rural o de pequeña localidad. En tales circunstancias uno puede poner en duda lo "urbanizado" 18 de la población. Podemos preguntarnos quién "socializa" a quién: los urbanos a los migrantes (con amplios antecedentes rurales), o es que los migrantes y sus hijos, al conservar e incrementar en los centros metropolitanos su comportamiento y sus valores propios de una sociedad agraria, predominan sobre los nativos dada su mayor proporción en la población de la ciudad. ${ }^{19}$

Por último, quisiéramos recalcar no sólo la complejidad situacional de las diferencias entre nativos y migrantes, sino su variabilidad a través del tiempo. La distinción nativo-migrante en el año 2000 en Monterrey puede ser muy diferente a la de 1965. México estará en otra etapa de desarrollo económico y la combinación nativo-migrante de la metrópoli reflejará este cambio.

18 Utilizado aquí en términos de los concomitantes socio-psicológicos de la existencia urbana, elaborado por Louis Wirth en su artículo de gran influencia, "Urbanism as a Way of Life", American Journal of Sociology, XLIV, julio de 1938

19 Esto podría explicar, por ejemplo, la aparente anomalía en América Latina de que la fecundidad urbana tiende a aumentar y a alcanzar tasas similares a la rural, y no lo contrario como sería de esperar de acuerdo con la teoría de la transición demográfica. Sobre México, véase Alvan 0 . Zárate, "Fertility in Urban Areas of Mexica: Implications for the Theory of the Demographic Transition", Demography, Vol. 4, Núm. 1, 1967, pp. 363-373; y su Community of Origin, Migration and Completed Marital Fertility in Metropolitan Monterrey, documento presentado en la reunión anual de la Population Association of America, Cincinatti, Ohio, abril de 1967. Esto también ayudará a explicar el hecho de que respecto a las preguntas sobre orientación de valores, en la encuesta de Monterrey, y que abarcan áreas tales como familia (roles del esposo, esposas y niños, aceptación del divorcio, limitación de la familia) hubiese variaciones relativamente pequeñas entre las seis categorías de migrantes, y bastante menores que las variaciones respecto a los 4 índices socioeconómicos. Tal vez el comportamiento en Monterrey en los valores predominantes se derive principalmente de fuentes rurales más que urbanas. 